\title{
水洜水轮机洜工况下近设计点驼峰现象的 流动机理研究*
}

阳 君 ${ }^{1,2,3}$ 袁寿其 $^{4}$ PAVESI Giorgio ${ }^{5}$ 李 春 ${ }^{1,2}$ 叶 舟 ${ }^{1,2}$

(1. 上海理工大学能源与动力工程学院 上海 200093;

2. 上海理工大学流动控制与仿真重点实验室 上海 200093;

3. 西华大学流体及动力机械教育部重点实验室 成都 610039;

4. 江苏大学国家水葲及系统工程技术研究中心 镇江 212013;

5. 帕多瓦大学工业工程系 帕多瓦 35131 意大利)

摘要: 中高比转速水泵水轮机原工况下近设计点驼峰严重制约了其稳定运行范围, 是制约抽水蓄能电站安全与经济运行的关 键问题之一。作为流动问题的宏观表现, 驼峰现象必然与水洜水轮机内部的非定常流动存在密切关系。为此, 基于试验和数 值模拟, 对水洜水轮机洜工况下可调导叶流道内的非定常流动进行研究, 探究模型机泵工况下的压力脉动特性、非定流动机 理及与性能曲线稳定性之间的关系。结果表明: 在设计和小于设计工况下, 可调导叶流道内均存在两种显著的周期性压力脉 动。模型机原工况下的流量扬程曲线在 $0.45 \sim 0.75$ 倍设计流量区间内出现驼峰, 频域分析清晰地揭示了以上两种压力脉动是 对该驼峰存在重要影响的流动参量。

关键词: 水泵水轮机; 原工况; 驼峰; 近设计点; 非定常流动

中图分类号: TH318

\section{Study of Hump Instability Phenomena in Pump Turbine at Large Partial Flow Conditions on Pump Mode}

\author{
YANG Jun $^{1,2,3}$ YUAN Shouqi $^{4} \quad$ PAVESI Giorgio $^{5} \quad$ LI Chun $^{1,2}$ YE Zhou ${ }^{1,2}$
}

(1. School of Energy and Power Engineering, University of Shanghai for Science and Technology, Shanghai 200093;

2. Key Laboratory of Flow Control and Simulation, University of Shanghai for Science and Technology, Shanghai 200093;

3. Key Laboratory of Fluid and Power Machinery of Ministry of Education, Xihua University, Chengdu 610039;

4. National Research Center of Pumps, Jiangsu University, Zhenjiang 212013;

5. Department of Industrial Engineering, University of Padua, Padua, 35131 Italy)

\begin{abstract}
For middle- and high- specific speed pump turbines, the hump instability appearing at large partial flow conditions seriously restricts its stable operation range. These phenomena relate closely to the inner unsteady flow in pump turbine. We investigate the characterization of pressure fluctuation and the mechanism of unsteady flow by an experimental and numerical study of the unsteady flow occurring among the adjustable blades. For pump working at either full or part load conditions, two kinds of periodic pressure pulsations in the diffuser vane have been observed and analyzed. A hump instability appears only when the actual flow rate of head curve is $0.45-0.75$ times of the designed value, and our frequency analysis further shows that its appearance is closely related to the two above pressure fluctuations.
\end{abstract}

Key words: pump turbine; pump mode; hump instability; large partial flow conditions; unsteady flow pattern

\section{0 前言}

对于水葲水轮机，其泵工况下的驼峰问题无法

* 国家自然科学基金青年基金(51506126)、上海理工大学流动控制与仿 真重点实验室(D15013)和流体及动力机械教育部重点实验室(重点研 究基地)开放课题(szjj2015-024) 资助项目。20151231 收到初稿, 20160619 收到修改稿
避免。驼峰是一种与机体结构及其内部流动密切相 关的反映在性能曲线上的一种不稳定现象。该问题 不仅影响水票水轮机洜工况高扬程条件下的起动和 运行，同时还会产生较强的振动和噪声，直接影响 机组安全稳定运行 ${ }^{[1-4]}$ 。目前, 该问题已成为制约水 泵水轮机发展的瓶颈和挑战。

水泵水轮机在泵工况下工作时可视作导叶式 
离心原。研究表明: 一方面, 在小流量时, 叶轮进 口回流作用易使流量-扬程曲线出现驼峰; 另一方 面, 当比转速大于 110 时, 其性能曲线还易在略小 于设计流量点附近出现驼峰 ${ }^{[5]}$, 即双驼峰。随着比 转速的增大, 该驼峰发生的风险也越高, 并且其发 生位置也将更接近设计流量 ${ }^{[6]}$ 。此外, 当驼峰出现 时还常伴随着迟滞效应, 如图 1 所示 ${ }^{[7]}$ 。因此, 近 设计点的驼峰与其伴随迟滞效应的出现严重制约了 中高比转速水洜水轮机运行的灵活性及其稳定工作 范围。

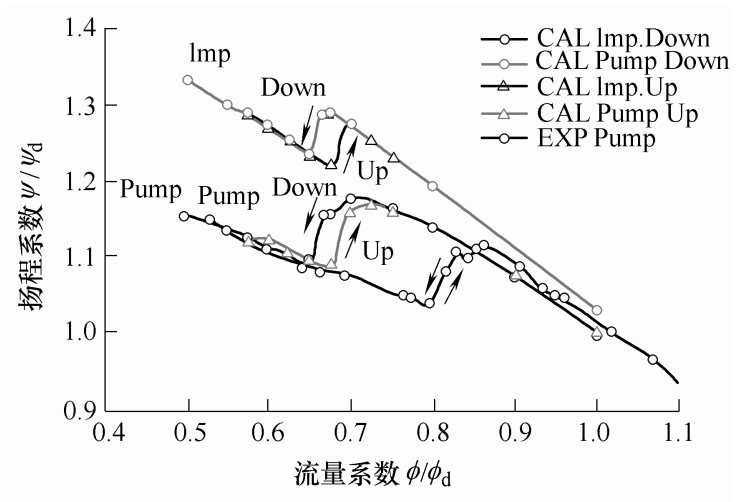

图 1 离心泵的磁滞效应

目前, 水葲水轮机洜工况下, 针对小流量驼峰 问题的研究(如发生机理、主动与被动抑制方法等), 业界学者已开展了较为深入、系统的工作 ${ }^{[8-13]}$, 但 针对近设计点驼峰产生机理的研究尚处在起步 阶段。

文献[14-15]通过试验及数值模拟方法对水洜 水轮机原工况下的双驼峰进行了研究, 指出叶轮进 出口区域的流态和驼峰的形成密切相关, 并在近设 计点驼峰区发现由旋转失速引起的低频压力脉动, 其频率为 0.2 倍叶轮转频。文献[16-19]分别采用多 种湍流模型对水洜水轮机洜工况下驼峰区的内部流 动进行数值预测研究。这些研究表明: 叶轮及扩散 段的非定常流动(如旋转失速、二次流、回流及浴结 构等) 是引起水泵水轮机泵工况驼峰问题的主要 原因。

尽管上述学者通过试验及数值模拟方法对水 泵水轮机近设计点的驼峰问题开展了研究, 但因水 洜水轮机在该区域时的流动极其复杂, 对于引起该 现象的成因尚未形成统一的解释。本文通过对水洜 水轮机泵工况下流场特征量的时域与频域分析, 获 得较为全面的非定常流动特征, 如压力脉动的周期 频率、作用强度等, 并采用流场可视化技术及计算 流体力学软件获得水百水轮机泵工况下流场的分布 规律。基于以上方法获得的结果, 得到水泵水轮机
泵工况下内部非定常流动特征及其与近设计点驼峰 之间的关系。为提出改善水泵水轮机驼峰问题提供 理论基础和技术支撑。

\section{1 试验装置}

本试验在意大利帕多瓦大学工业工程系开式 旋转机械试验台(Open turbomachinery facility, OTF)上进行, 试验台如图 2 所示。该试验台按照国 际标准 IEC60041 及 IEC60193 设计, 专用于测试旋 转水力机械(水泵、水轮机及水泵水轮机等)的水力 性能及其瞬态特性, 如压力、振动和声压等。

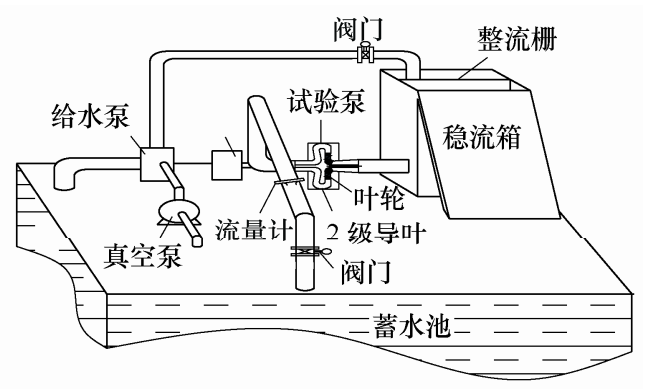

(a) 示意图

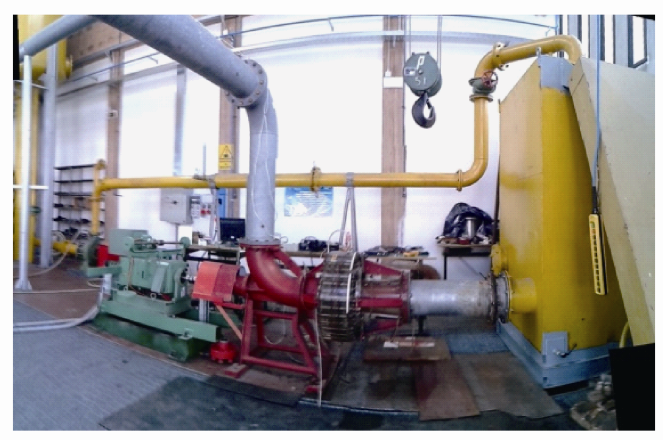

(b) 实物图

图 2 开式旋转机械试验台

试验研究对象为一个二级水百水轮机的低压 级。转速为 $600 \mathrm{r} / \mathrm{min}$, 比转速为 137.24 (易发生双驼 峰现象), 其结构如图 3 所示, 主要由壳体、叶轮和 回流腔组成。回流腔用于引导叶轮出口的流体进入 下级, 由两级导叶组成: 22 个可调导叶及 11 个 $\mathrm{U}$ 形弯曲固定导叶。叶轮与可调导叶进口的径向间隙 为 $5 \mathrm{~mm}$ (2.5\%叶轮半径)。主要参数如表 1 所示: 表 中 $D_{2} 、 D_{3}$ 和 $D_{4}$ 分别为叶轮、可调导叶及固定导叶 叶片的直径, $B_{2} 、 B_{3}$ 和 $B_{4}$ 分别为叶轮、可调导叶及 固定导叶出口宽度, $n_{\mathrm{b} 2} 、 n_{\mathrm{b} 3}$ 和 $n_{\mathrm{b} 4}$ 分别为叶轮、可 调导叶及固定导叶叶片数, $\beta_{2 \mathrm{c}}$ 为叶轮叶片出口角, $\phi_{\text {Des }}$ 为模型设计点流量系数, $\alpha_{3 \mathrm{c}}$ 和 $\alpha_{4 \mathrm{c}}$ 分别为可调 导叶及固定导叶出口水流角, $\lambda$ 表示可调导叶及固 定导叶之间的周向方位角为 $8^{\circ}$ 。根据以上参数可 
知, 叶片通过频率 $f_{\mathrm{BPF}}=70 \mathrm{~Hz}$ 。本文研究工况为该 模型在可调导叶出口水流角为 $18^{\circ}$ 时的泵工况。

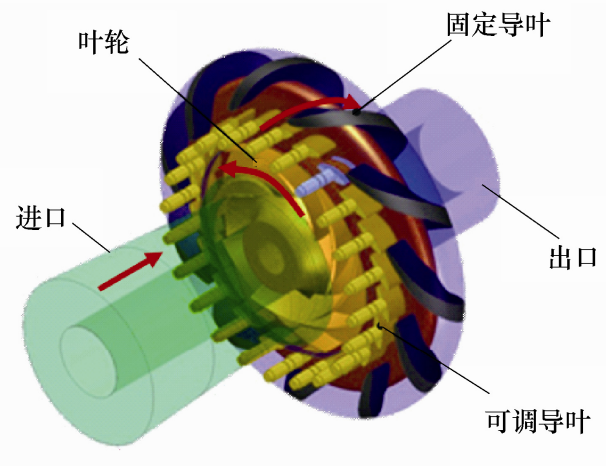

液体介质流动方向 $\longrightarrow$

图 3 二级水泵水轮机低压级模型

表 1 水蜃水轮机模型主要参数

\begin{tabular}{ccc}
\hline & 参数 & 数值 \\
\hline & 直径 $D_{2} / \mathrm{mm}$ & 400 \\
& 宽度 $B_{2} / \mathrm{mm}$ & 40 \\
叶片数 $n_{\mathrm{b} 2}$ & 7 \\
叶轮参数 & 出口角 $\left.\beta_{2 \mathrm{c}}{ }^{\circ}\right)$ & 26.5 \\
& 点流量系数 $\phi_{\mathrm{Des}}$ & 0.125 \\
\hline & 直径 $D_{3} / \mathrm{mm}$ & 410 \\
& 宽度 $B_{3} / \mathrm{mm}$ & 40 \\
可调导叶参数 & 叶片数 $n_{\mathrm{b} 3}$ & 22 \\
& 可调导叶出口水流角 $\alpha_{3 \mathrm{c}} /\left(^{\circ}\right)$ & 18 \\
& 周向方位角 $\lambda /\left({ }^{\circ}\right)$ & 8 \\
\hline & 直径 $D_{4} / \mathrm{mm}$ & 516 \\
宽度 $B_{4} / \mathrm{mm}$ & 40 \\
固定导叶参数 & 叶片数 $n_{\mathrm{b} 4}$ & 11 \\
& 固定导叶出口水流角 $\alpha_{4 \mathrm{c}} /\left(^{\circ}\right)$ & 30 \\
\hline
\end{tabular}

模型机的试验主要分为以下三部分: (1) 水力 性能测量; (2) 可调导叶表面动态压力测量; (3) 流 场可视化测量。在水力性能测量时, 进、出口压力 分别采用相对压力传感器 WIKA891.12.500 和绝对 压力传感器 PHILIPS 940421560161 测量; 流量采 用根据 ASME PTC 19.5-2004 校准的喷嘴流量计测 量; 扭矩测量采用 Kistler 4503A 扭矩仪测量。

已有研究表明: 近设计点的驼峰与导叶流道内 的非定常流动密切相关。因此, 本试验采用 12 枚 XCL-072 系列小型 IS 压力传感器对可调导叶表面 瞬态压力进行测量, 其安装示意图如图 4 所示。

为定性描述和分析二级导叶流道内的非定常 流动, 本文采用高压气泡作为流道内部流动的示踪 媒介, 高压气泡的注入孔位置如图 5 所示。通过高 速摄影仪拍摄示踪气泡在流道内的流动轨迹, 以实 现流场可视化的测量。其中, 所采用的高速摄影仪 为 Photron FASTCAM PCI 数字摄像机, 记录图片的 分辨率为 512 像素 $\times 512$ 像素, 拍摄采用 5000 帧/s。

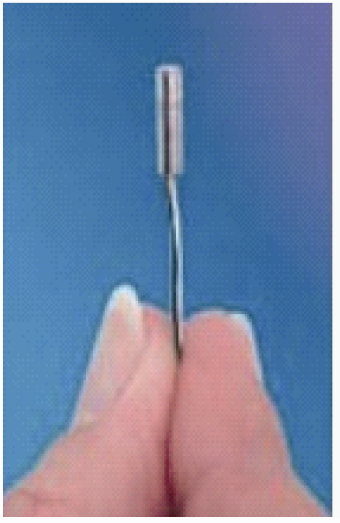

(a) IS 压力传感器

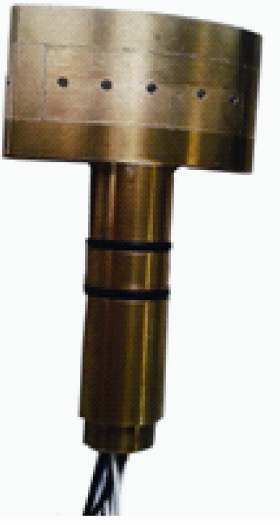

(b) 可调导叶片

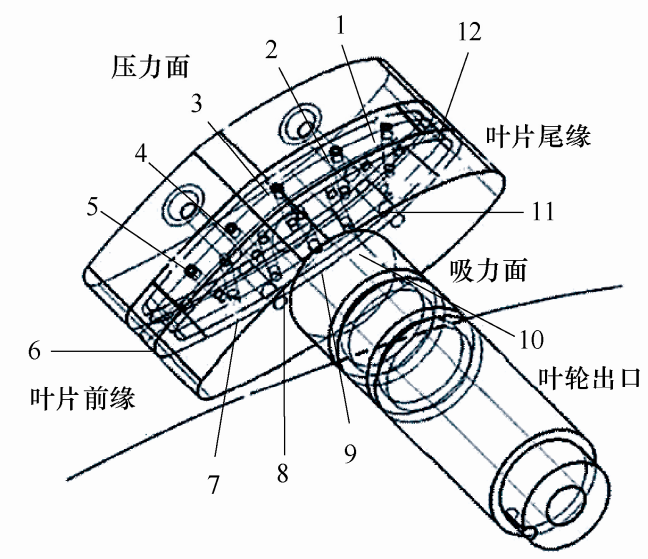

(c) 安装示意图

图 4 可调导叶及其动态压力传感器的安装

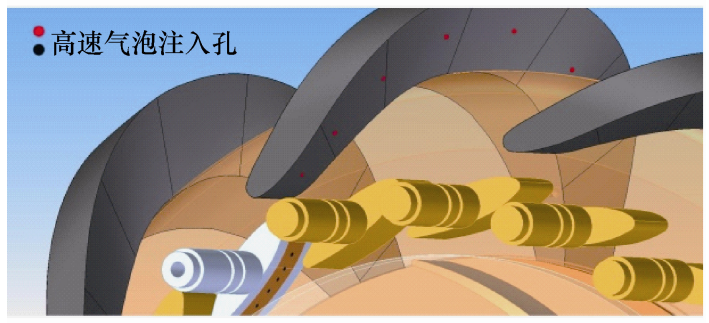

图 5 导叶表面高速气泡注入孔

\section{2 信号处理}

在压力脉动的频谱分析中, 叶片通过频率为频 域中的显著特征频率之一, 文中引入斯特劳哈尔数 $(S t)$ 对频率进行归一化处理, 该数基于周向叶片间隙 和叶片叶尖转速定义如下所示 ${ }^{[20]}$

$$
S t=\frac{\pi D_{2}}{n_{\mathrm{b}}} \frac{f}{u_{2}}=\frac{f}{f_{\mathrm{BPF}}}
$$

式中, $D_{2}$ 为叶轮直径; $n_{\mathrm{b}}$ 为叶轮叶片数; $u_{2}$ 为叶轮 出口切向速度。

根据奈奎斯特采样定律: 在进行模拟/数字信号 的转换过程中, 当采样频率 $f_{\mathrm{s}}$ 大于信号中最高频率 $f_{\text {max }}$ 的 2 倍时 $\left(f_{\mathrm{s}}>2 f_{\text {max }}\right)$, 采样之后的数字信号完整地 
保留了原始信号中的信息。一般实际应用中保证采 样频率为信号最高频率的 $5 \sim 10$ 倍 ${ }^{[21]}$ 。本文主要研 究叶片通过频率附近的非定常流动, 因此试验压力 采样率应至少大于 2 倍叶片通过频率 $(140 \mathrm{~Hz})$, 本 试验中压力信号采样频率为 $2^{10} \mathrm{~Hz}$, 采样时长 $2^{8} \mathrm{~s}$ 。

目前, 国内对旋转水力机械压力脉动频谱分析 主要采用快速傅里叶变换(Fast Fourier transform,

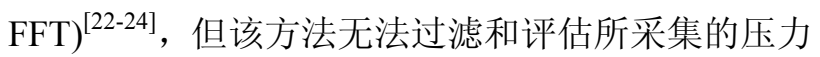
数据中由系统、环境等引起的随机信号误差干扰。 此外, 本试验中各种随机过程对所采集的动态压力 数据的影响是不可忽略的, 故本文采用功率谱密度 估计法对所测模型机内部压力脉动进行分析。该方 法为一种概率统计方法, 是对随机变量均方值的量 度, 连续瞬态响应通过概率分布函数进行描述, 即 出现某水平响应所对应的概率 ${ }^{[25]}$ 。

根据维纳-辛钦定理(Wiener-Khinchin theorem), 随机离散随机过程 $x[n]$, 其功率谱密度与其自相关 函数为傅里叶变换 ${ }^{[26]}$

$$
G_{x x}(f)=\sum_{k=-\infty}^{\infty} r_{x x}[k] \exp (-\mathrm{j} 2 \pi k f)
$$

式中, $r_{x x}[k]=E\left[X[n] x^{*}[n-k]\right]$ 为其自相关函数。本 试验中 $x[n]$ 为试验采样得到的压力脉动数据离散时 间序列, 功率谱密度可写成

$$
G_{x x}(f)=\lim _{T \rightarrow \infty} E\left[\frac{1}{T}\left|X(f)_{T}\right|^{2}\right]
$$

式中, $T$ 为数据采样时间, $X(f)$ 为 $x[n]$ 的傅里叶变换。

工程应用中, 经典谱估计方法得到了广泛的应 用, 本文采用经典谱估计方法的改进方法, 即 Welch 法, 对所测压力脉动进行频谱分析。Welch 法是标 准周期法和 Bartlett 法的结合, 其优点在于对窗函数 没有特殊要求, 任何窗函数均可以使谱估计非 负 ${ }^{[27]}$ 。同时, 分段时为减少由于分段数增加给分辨 率带来的影响, 采用各段之间均重叠的方法处理, 其步骤如下。

(1) 将采样数据分为 $N$ 段, 每段数据长度为 $L$, 数据重叠率为 $D$ 。

（2）选择适当的窗函数 $w[n]$, 对每段数据依次 加权, 然后确定每段的周期图。

(3) 对各段的周期图进行平均得到功率谱估。

本试验采用汉宁窗(Hanning window)对每段数 据进行加权, 其中 $N$ 为 $2^{8}, L$ 为 $2^{13}, D$ 为 $50 \%$, 其自功率谱写为 ${ }^{[28]}$

$$
G_{x x}(f)=\frac{1}{N W_{\mathrm{H}}} \sum_{k=1}^{N}\left[X_{k}^{*}(f) X_{k}(f)\right]
$$

式中, $k$ 为第 $k$ 段数据; $W_{\mathrm{H}}$ 为对应汉宁窗的加权系
数; $X_{k}(f)$ 为压力信号 $x$ 第 $k$ 段快速傅里叶变换; $X_{k}^{*}(f)$ 为函数 $X_{k}(f)$ 的复共轭。

同时, 为分析各压力信号之间频率分量的关联 度, 本文对不同压力监测点的信号进行互功率谱密 度分析, 其互功率谱(Cross-spectra) 写为 ${ }^{[28]}$

$$
G_{x y}(f)=\frac{1}{N W_{\mathrm{H}}} \sum_{k=1}^{N}\left[X_{k}^{*}(f) Y_{k}(f)\right]
$$

式中, $G_{x y}(f)$ 表示信号 $x$ 和 $y$ 的功率谱密度。 $G_{x y}(f)$ 越大, 表明两个信号 $x$ 和 $y$ 的相应频率分量关联度很 高, 如果 $G_{x y}(f)=0$, 表明其相应频率分量是正交的。

\section{3 结果分析}

\section{1 模型机性能分析}

图 6 为模型机在泵工况下的试验性能曲线。由 图可知, 该模型机在泵工况下性能曲线出现双驼峰 现象: 不仅在小于 $0.40 Q_{\text {Des }}\left(Q_{\text {Des }}\right.$ 为模型机原工况下 设计流量)时出现驼峰, 还在 $0.45 Q_{\mathrm{Des}} \sim 0.75 Q_{\mathrm{Des}}$ 时, 出现驼峰并伴随磁滞效应, $0.6 Q_{\text {Des }}$ 附近为近设计点 驼峰的扬程极值点。

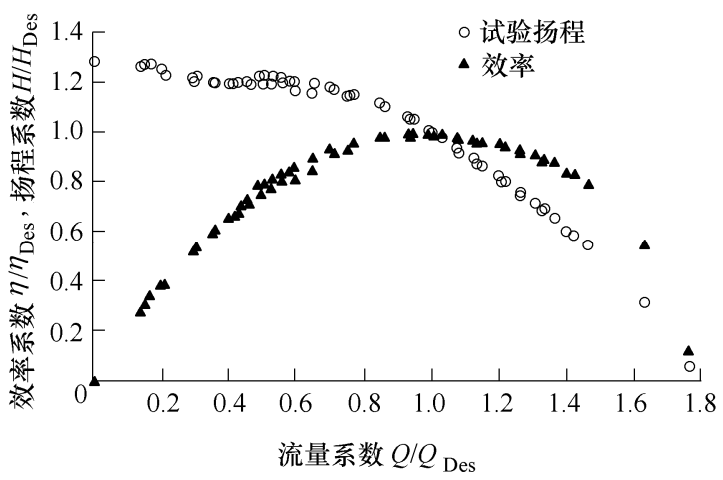

图 6 模型水葲水轮机葲工况性能曲线

\section{2 设计工况下压力脉动分析}

为得到模型机在洜工况下非定常流动的特征, 通过 Welch 频谱估计法对所测可调导叶表面压力脉 动进行处理, 得到压力监测点频谱图, 图 7 为设计
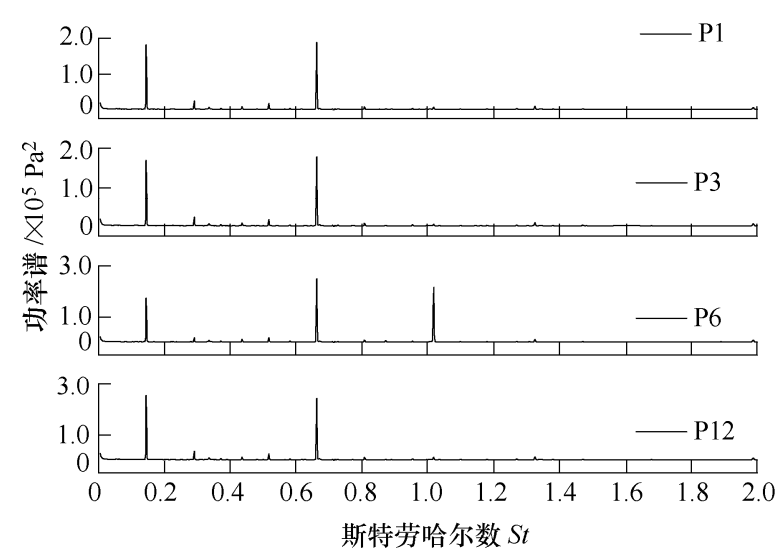

图 7 设计流量压力监测点自功率谱图 
流量下可调导叶表面监测点 1、3、6 和 12(各点位 置见图 4)的自功率谱图。

由于叶轮与导叶的动静干涉作用, 各监测点在 轴频 $S t=0.143\left(f_{\mathrm{BPF}} / n_{\mathrm{b} 2}, 10 \mathrm{~Hz}\right)$ 和叶片通过频率 $S t=1\left(f_{\mathrm{BPF}}, 70 \mathrm{~Hz}\right)$ 及其谐频处出现峰值。

同时由图 7 可知, 在设计流量下, 除了受到动 静干涉的影响外, 在 $S t=0.6625\left(0.6625 f_{\mathrm{BPF}}, 46.375\right.$ $\mathrm{Hz}$ )也出现显著峰值, 该频率与叶轮转速及叶片数 无关。

为了分析各监测点压力信号之间的频率分量 $S t=0.143 、 S t=0.6625$ 和 $S t=1$ 的关联度, 本文对各监 测点压力信号进行互功率谱分析。图 8 为部分监测 点在设计流量下的互功率谱图。
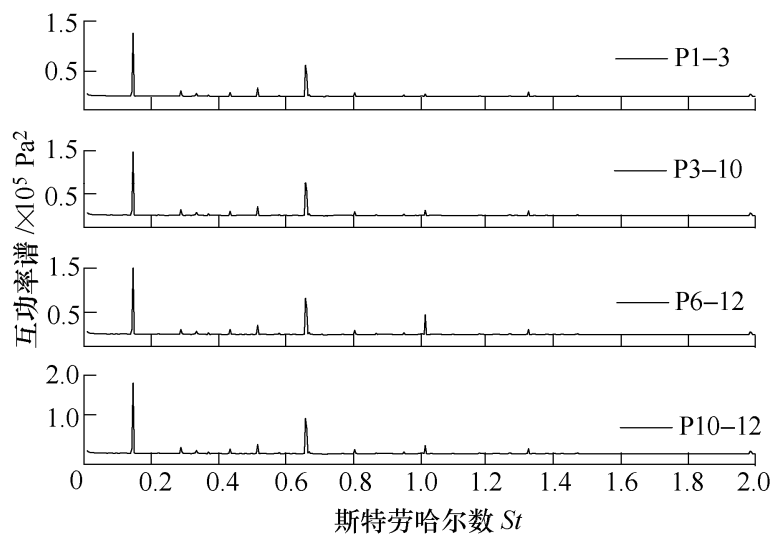

图 8 设计流量压力监测点互功率谱图

由图 8 可知, 可调导叶各监测点的压力脉动均 受到叶片通过频率的影响, 但随着离叶轮距离的加 大而影响减弱。监测点 6 最靠近叶轮, 因此每个叶 片扫过该监测点的作用显著, 即在叶片通过频率 $S t=1$ 处的峰值最为显著。随着监测点远离叶轮出口, 监测点受到该作用的影响减弱, 使得监测点 $1 、 3 、$ 12 的压力自功率谱中该处的峰值较小。

因此, 在设计流量下可调导叶流道内, 以下两 种周期性压力脉动最为显著: 特征频率为轴频 $S t=0.143$ 的周期性压力脉动; 与转速不相关的特征 频率为 $S t=0.6625$ 的周期性压力脉动。

\section{3 不同流量下压力脉动分析}

为研究以上两种周期性压力脉动在不同流量下 的变化特征, 并探索其与近设计点附近出现驼峰之 间是否存在关系, 对 $0.3 Q_{\text {Des }} \sim 1.2 Q_{\text {Des }}$ 流量范围内不 同流量下的压力脉动的自功率谱进行分析。图 9 为 压力监测点 5、6、11 和 12 在该流量范围内的自功 率谱。

由图可知, 在该流量范围内, 导叶流道内均出 现以轴频 $S t=0.143$, 和 $S t=0.6625$ 为特征频率的压 力脉动。

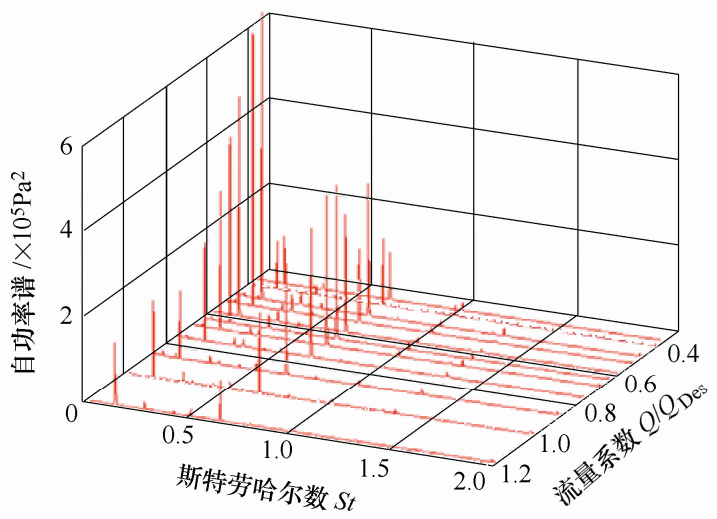

(a) 监测点 5

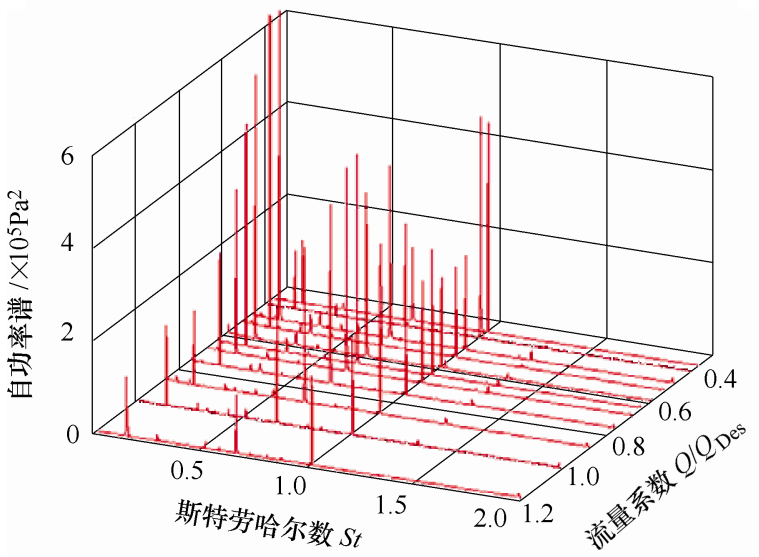

(b) 监测点 6

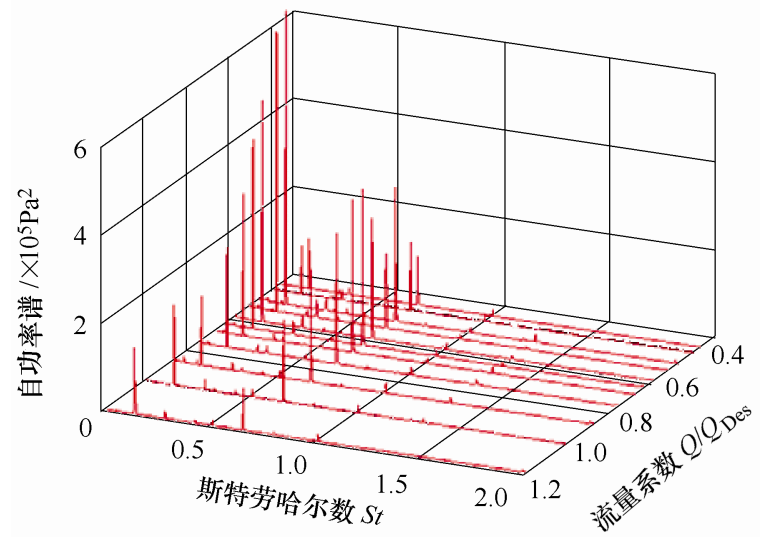

(c) 监测点 11

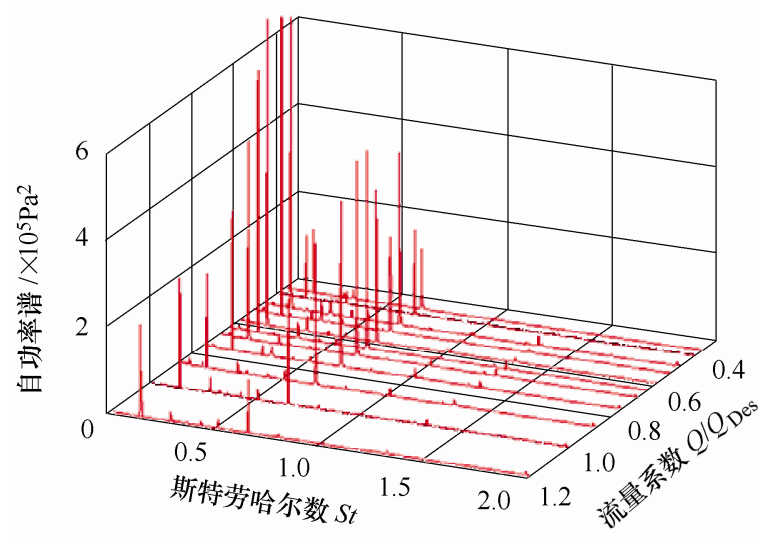

(d) 监测点 12

图 9 压力监测点 5、6、11 和 12 在不同流量下的自功率谱 
以 $S t=0.143$ 为特征频率的压力脉动, 在大流量 和设计流量附近 $\left(0.8 Q_{\mathrm{Des}} \sim 1.2 Q_{\mathrm{Des}}\right)$ 幅值变化不大, 随着流量进一步减小, 其脉动幅值开始逐渐增大。 当流量进入近设计点驼峰区, 其幅值增幅最显著。

从大流量到近设计点的驼峰区 $\left(1.2 Q_{\text {Des }} \sim\right.$ $0.45 Q_{\text {Des }}$ ), 频率 $S t=0.6625$ 压力脉动幅值随流量变 化的趋势, 虽然与 $S t=0.143$ 处的变化趋势不同, 但 与 PAVESI 等试验测出的该模型机叶轮出口压力随 流量变化的趋势是一致, 如图 10 所示 ${ }^{[14]}$ 。在 $1.2 Q_{\text {Des }} \sim 0.8 Q_{\text {Des }}$ 范围内, 随着流量的减小压力幅值 缓慢增加, 当流量进入所关注驼峰区时, 压力幅值 随流量减小显著增加。在 $0.6 Q_{\text {Des }}$ 附近幅值最大, 随 后幅值出现突降。

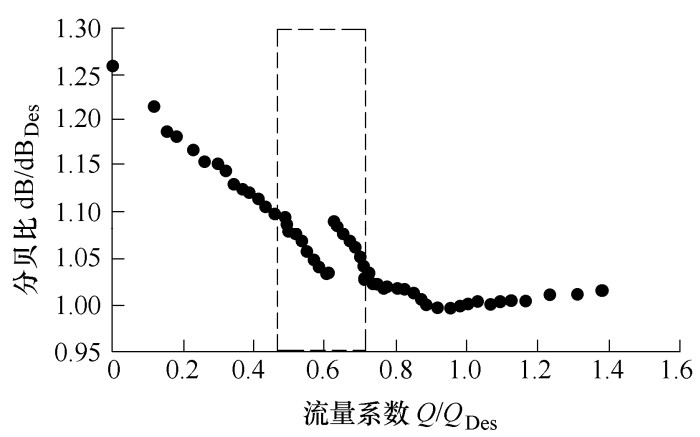

图 10 叶轮出口平均压力随流量的变化

\section{$3.4 S t=0.6625$ 特征频率压力脉动机理探究}

图 11 为文献[26]中对模型机进行流场预测得到 的设计流量下原内某一时刻不同截面的流场分布, 其不同颜色代表流速大小。在设计流量下, 叶轮及 可调导叶流道内流动均未出现明显的非定常流动。 但在固定导叶流道内, 由于其 $\mathrm{U}$ 形扭曲流道, 在靠 近前盖板附近出现较为明显的非定常流动。同时由 图 8 可知, 试验中可调导叶流道内在设计流量下出 现特征频率为 $S t=0.6625$ 压力脉动。由此推断：该 特征频率的压力脉动与上述固定导叶流道内的非定 常流动存在一定联系。试验所得可视化流场结果也 同时验证了该推断。如图 12 所示, 设计流量下, 可 调反导叶流道内的示踪气泡流动较为平稳, 即与模 拟结果相吻合。在固定反导叶流道内, 在吸力面前 缘附近注入气泡, 气体从注入孔进入流场后便被流 道内的来流快速打散成为细小的螺旋状颗粒气泡 云, 并在该气泡云中发现 $S t=0.6625$ 的周期性波动。 该结果证实了: U 形固定导叶吸力面附近的非定常 流动是引起可调导叶中特征频率为 $S t=0.6625$ 压力 脉动的原因。

本文对模型机流场数值模拟结果中导叶内压 力场进行频域分析后的结果也与试验相符。叶轮及 导叶中截面在 $S t=0.6625$ 下的压力分布如图 13 所

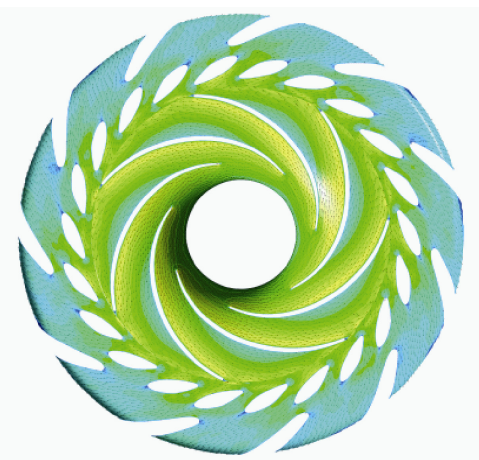

(a) 近后盖板轴截面

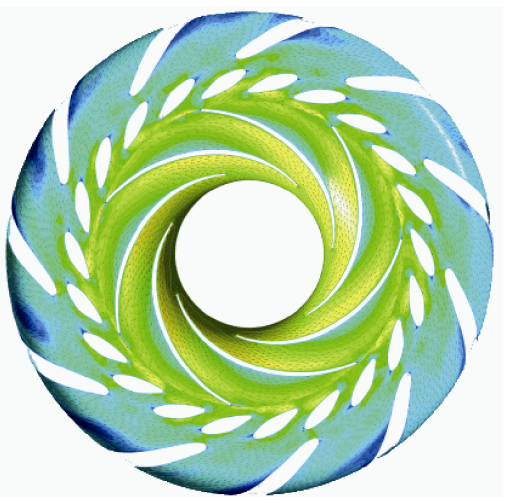

(b) 中截面

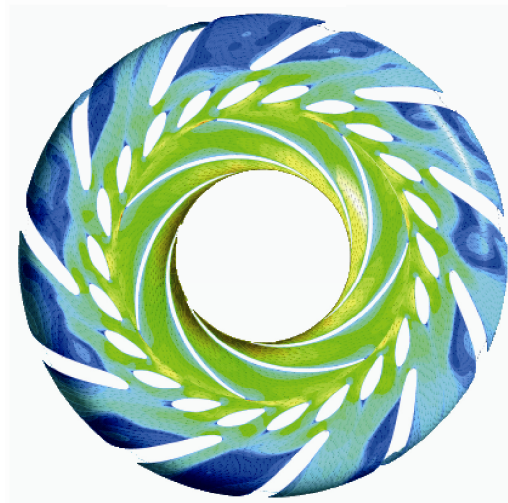

(c) 近前盖板轴载面

图 11 设计流量下叶轮及回流腔不同轴截面流场图

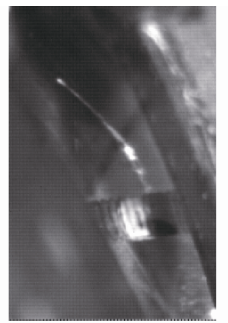

(a) 可调导叶流道

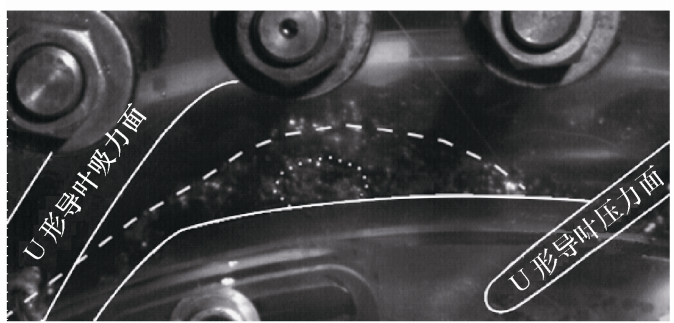

(b) 固定导叶流道

图 12 高速摄影仪所拍设计流量下导叶流道内流场 
示, 设计流量该频率下的压力高幅值区域主要出现 在 $U$ 形导叶吸力面附近及可调导叶流道, 与试验 结果吻合。同时该图进一步说明: 随着流量的减 小, 源于 $\mathrm{U}$ 形固定导叶内特征频率 $S t=0.6625$ 的压 力脉动对可调导叶流动内的作用显著, 如图 $13 \mathrm{~b}$ 所示。

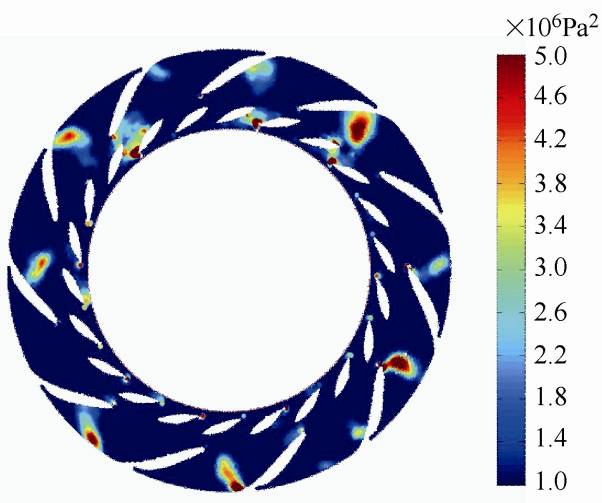

(a) $1.0 Q_{\text {Des }}$

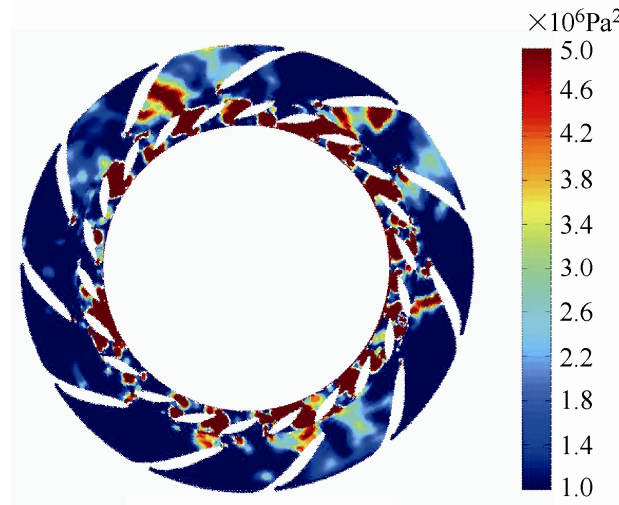

(b) $0.584 Q_{\mathrm{Des}}$

图 13 设计流量及 0.584 倍设计流量回流腔中截面上压力在 频率 $S t=0.6625$ 下幅值分布图

综合不同流量下的压力脉动分析可知: 可调导 叶流道内特征频率为 $S t=0.6625$ 压力脉动, 在模型 泵运行工况初入近设计点驼峰区 $\left(0.45 Q_{\text {Des }} \sim\right.$ $0.75 Q_{\text {Des }}$ ) 时, 幅值增加显著, 并在 $0.6 Q_{\text {Des }}$ 附近出 现最大值。随着流量的进一步减小, 模型机叶轮中 的流动分离、射流尾迹等非定常流动逐渐显著, 使 得其下游可调导叶内流动更加复杂。这有可能是导 致 $0.6 Q_{\text {Des }}$ 附近该特征频率下的压力幅值突降的 原因。

\section{4 结论}

(1) 模型机可调导叶流道内在两个驼峰区均出 现显著的特征频率为轴频 $S t=0.143$ 和 $S t=0.6625$ 的 周期性压力脉动, 以上两种压力脉动对近设计点驼 峰存在重要影响。其中, 特征频率为轴频的压力脉
动主要由叶轮导叶动静干涉引起, 即动静干涉作用 对双驼峰都具有一定的影响。

(2) 由于模型机的 U 形扭曲固定导叶的设计, 使其在葲工况运行过程中，即使是设计流量下，其 固定导叶流道内也出现特征频率与叶片通过频率及 轴频无关的非定常流动结构, 其频率值为 0.6625 倍 $f_{\mathrm{BPF}}$, 即 $S t=0.6625$ 。

(3) 得出 $U$ 形扭曲固定导叶引起的 $S t=0.6625$ 流动结构是引起可调导叶内该频率压力脉动的原 因, 并对可调导叶流道和叶轮出口的流场都有一定 影响。同时可调导叶内该频率的压力脉动是引起近 设计点驼峰的主要原因之一。随着流量的减小，其 影响加剧, 并在近设计点驼峰的扬程极值点 $\left(0.6 Q_{\text {Des }}\right)$ 附近作用最为显著, 随后开始随着流量减小而减小， 其原因将在后续研究中进一步探究。

\section{参 考 文 献}

[1] 傅之跃, 刘伟超, 郑津生, 等. 东方电机水泵水轮机水 力开发的技术进步 $[\mathrm{J}]$. 水电站机电技术, 2010, 25(2): 5-9.

FU Zhiyue, LIU Weichao, ZHENG Jinsheng, et al. Technical progress of hydraulic development for pumpturbine in DFEM[J]. Mechanical \& Electrical Technique of Hydropower Station， 2010， 25(2): 5-9.

[2] 马鹏飞, 王军, 王俊, 等. 高比转速双向原马鞍区流动 特性分析 [J]. 流体机械, 2014, 42(10): 11-15.

MA Pengfeng, WANG Jun, WANG Jun, et al. Flow characteristics analysis in saddle zone for high specific speed bi-directional pump[J]. Fluid Machinery, 2014, 42(10): 11-15.

[3] 梅祖彦. 抽水蓄能发电技术 $[\mathrm{M}]$. 北京: 机械工业出版 社, 2000 .

MEI Zuyan. Pumped storage power generation technology [M]. Beijing: China Mechine Press, 2000.

[4] LI Deyou, WANG Hongjie, XIANG Gaoming, et al. Unsteady simulation and analysis for hump characteristics of a pump turbine model[J]. Renewable Energy, 2015, 77: $32-42$

[5] HERGT P, STARKE J. Flow patterns causing instabilities in the performance curves of centrifugal pumps with vaned diffusers[C]/Proceedings of the Second International Pump Symposium, 1985: 67-75.

[6] GÜLICH J F. Centrifugal pumps [M]. London: Springer, 2010.

[7] IINO M, TANAKA K, MIYAGAWA K, et al. Numerical simulation of hysteresis on head/discharge characteristics 
of a centrifugal pump[C]//ASME/ JSME 2003 4th Joint Fluids Summer Engineering Conference, 2003, 1165-1172.

[8] MURAKAMI M, HEYA N. Swirling flow in suction pipe of centrifugal pumps: 1st report, distribution of velocity and energy [J]. Bulletin of JSME, 1966, 34 (9): 328-337.

[9] BRENNEN C, ACOSTA A. The dynamic transfer function for a cavitating inducer[J]. Journal of Fluids Engineering, 1976, 98(2): 182-191.

[10] GREITZER E M. The stability of pumping systems - the 1980 Freeman Scholar Lecture[J]. Journal of Fluids Engineering, 1981, 103(2): 193-242.

[11] BREUGELMANS F, SEN M. Prerotation and fluid recirculation in the suction pipe of centrifugal pumps[C]//Proc 11th Int. Pump Symp, Texas A\&M Univ., 1982, 165-180.

[12] BARRAND J, CAIGNAERT G, CANAVELIS R, et al. Experimental determination of the reverse flow onset in a centrifugal impeller[C/CD]//Proceedings of the First International Pump Symposium Texas A\&M University, 1984.

[13] ZHANG Z. Rotating stall mechanism and stability control in the pump flows[C]//OP Conference Series: Earth and Environmental Science, 2010, 12(1): 012010.

[14] RAN H, LUO X, ZHU L, et al. Experimental study of the pressure fluctuations in a pump turbine at large partial flow conditions [J]. Chinese Journal of Mechanical Engineering, 2012, 25(6): 1205-1209.

[15] 冉红娟, 张瑶, 罗先武, 等. 可逆式水轮机原工况下驼 峰现象的数值模拟 $[\mathrm{J}]$. 水力发电学报, $2011,30(3)$ : 175-179.

RAN Hongjuan, ZHANG Yao, LUO Xianwu, et al. Numerical simulation of the positive-slope performance curve of a reversible hydro-turbine in pumping mode[J]. Journal of Hydroelectric Engineering, 2011, 30(3): 175-179.

[16] YAO Yangyang, XIAO Yexiang, ZHU Wei, et al. Numerical analysis of a model pump-turbine internal flow behavior in pump hump district[C]//IOP Conference Series: Earth and Environmental Science, 2014, 22(3): 032040 .

[17] 王焕茂. 混流式水百水轮机驼峰区数值模拟及试验研 究[D]. 武汉; 华中科技大学, 2009 .

WANG Huanmao. Francis pump turbine hump numerical simulation and experimental study[D]. Wuhan: Huazhong University of Science and Technology, 2009.

[18] 王焕茂, 吴钢, 吴伟章, 等. 混流式水百水轮机驼峰区
数值模拟及分析 $[\mathrm{J}]$. 水力发电学报, 2012, 31(6): 253-258.

WANG Huanmao, WU Gang, WU Weizhang, et al. Numerical simulation and analysis of the hump district of Francis pump-turbine[J]. Journal of Hydroelectric Engineering, 2012, 31(6): 253-258.

[19] 陶然, 肖若富, 杨魏, 等. 可逆式水泵水轮机原工况的 驼峰特性 [J]. 排灌机械工程学报, 2014, 32(11): 927-930.

TAO Ran, XIAO Ruofu, YANG Wei, et al. Hump characteristic of reversible pump-turbine in pump mode $[\mathrm{J}]$. Journal of Drainage and Irrigation Machinery Engineering, 2014, 32(11): 927-930.

[20] CAVAZZINI G. Rotor-stator interaction in radial turbomachines[M]. Saarbiüken: LAP Lambert Academic Publishing, 2013.

[21] 施卫东, 姚捷, 张德胜, 等. 采样频率和时间对轴流葲 压力脉动特性的影响 $[\mathrm{J}]$. 排灌机械工程学报， 2013， 31(3): 190-194.

SHI Weidong, YAO Jie, ZHANG Desheng, et al. Influence of sampling frequency and time on pressure fluctuation characteristics of axial-flow pump[J]. Journal of Drainage and Irrigation Machinery Engineering, 2013, 31(3): 190-194.

[22] 王乐勤, 刘迎圆, 刘万江, 等. 水泵水轮机泵工况的压 力脉动特性 [J]. 排灌机械工程学报, 2013, 31(1): 7-10. WANG Leqin, LIU Yingyuan, LIU Wanjiang, et al. Pressure fluctuation characteristics of pump-turbine at pump mode[J]. Journal of Drainage and Irrigation Machinery Engineering, 2013, 31(1): 7-10.

[23] 张金风，袁寿其，付跃登，等. 分流叶片对离心原流场 和性能影响的数值预报 [J]. 机械工程学报, 2009, 45(7): 131-137.

ZHANG Jinfeng, YUAN Shouqi, FU Yuedeng, et al. Numerical forecast of the influence of splitter blades on the flow field and characteristics of a centrifugal pump $[\mathrm{J}]$. Journal of Mechanical Engineering, 2009, 45(7): 131-137.

[24] 江伟, 李国君, 张新盛. 离心原叶片出口边倾斜角对压 力脉动的影响 $[\mathrm{J}]$. 排灌机械工程学报, 2013, 31(5): 369-372, 378 .

JIANG Wei, LI Guojun, ZHANG Xinsheng. Effect of oblique angle of blade trailing edge on pressure fluctuation in centrifugal pump[J]. Journal of Drainage and Irrigation Machinery Engineering, 2013, 31(5): 369-372, 378.

[25] 孙春艳, 徐伟. 分数阶导数阻尼下非线性随机振动结构 响应的功率谱密度估计 [J]. 应用力学学报, 2013, 
30(3): 401-404.

SUN Chunyan, XU Wei. Response power spectral density estimate of a fractionally damped nonlinear oscillator[J]. Chinese Journal of Applied Mechanics, 2013, 30(3): 401-404.

[26] YANG J, PAVESI G, CAVAZZINI G, et al. Numerical characterization of pressure instabilities in a vaned centrifugal pump under partload condition[C]// IOP Conference Series: Materials Science and Engineering, 2013, 52(2): 22-44.

[27] 李晓豁, 韩宇飞. 掘进机截割载荷极值能量的频率识
别[J]. 煤炭学报, 2008, 33(4): 459-461.

LI Xiaohuo, HAN Yufei. Frequency recognition of cutting loads extreme energy for roadheader[J]. Journal of China Coal Society, 2008, 33(4): 459-461.

[28] PAVESI G, CAVAZZINI G, ARDIZZON G. Timefrequency characterization of the unsteady phenomena in a centrifugal pump [J]. International Journal of Heat and Fluid Flow, 2008， 29(5): 1527-1540.

作者简介: 阳君, 女, 1987 年出生, 博士。主要研究方向为透平机械内 部流动及其流动诱导噪声。

E-mail: yangjun@usst.edu.cn 